\title{
Comparison of treatment outcomes in neck pain patients depending on the sex of the chiropractor: a prospective outcome study
}

\author{
Janine Thöni, Cynthia K. Peterson ${ }^{*}$ and B. Kim Humphreys
}

\begin{abstract}
Background: The high percentage of female chiropractic students in Switzerland suggests a future sex shift in the chiropractic profession in Switzerland. Thus the purpose of this study is to determine if male and female chiropractors achieve the same treatment outcomes in neck pain patients.

Methods: Included in this prospective outcomes study were 849 patients with neck pain of any duration. Prior to the first treatment, baseline demographic data, the Bournemouth Questionnaire (BQ) and the numerical rating scale (NRS) for neck and arm pain were completed. At the follow-up time points of 1 week, 1, 3, 6 and 12 months, the Patient's Global Impression of Change (PGIC) scale to categorize the actual 'improvement' and the BQ and the NRS for neck pain were completed.

The Chi-square test compared the proportion of patients reporting 'improvement' between male and female chiropractors for each time point. The unpaired Student's t-test compared the BQ and the NRS actual and change scores between patients of male and female chiropractors at all time points. Demographic factors were compared between the sexes using the Chi-square test.
\end{abstract}

Results: Proportionally more patients of female chiropractors reported 'improvement' at 1 month $(p=0.035)$ and significantly more pain reduction at 3 months $(p=0.040)$. Patients of male chiropractors presented with significant older age $(p=0.0001)$, higher levels of baseline neck pain $(p=0.012)$, a lower proportion with radiculopathy $(p=0.014)$ and less pain medication use $(p=0.046)$.

Conclusions: Female chiropractors achieve at least equally satisfying treatment results for neck pain patients compared to male chiropractors. Female chiropractors also have a higher proportion of female patients compared to male chiropractors and patients presenting with radiculopathy and using pain medications.

Trial registration: Not applicable for this type of study.

Keywords: Neck pain mechanical, Treatment outcome, Chiropractic, Spinal manipulative therapy, Gender

\section{Background}

From the Swiss Job Analysis Survey 2009 it is known that there is a strong male predominance in the chiropractic profession in Switzerland [1]. This was also said to be found in the job analysis reports published for the United Kingdom and the United States, showing that there are more men than women working as chiropractors [2-4]. The sex ratio for the whole Swiss Association of Chiropractors in 2009 was $27 \%$ female versus $73 \%$

\footnotetext{
* Correspondence: Cynthia.peterson@balgrist.ch; x-raydcpeterson@yahoo.ca Department of Chiropractic Medicine, Orthopaedic University Hospital Balgrist, University of Zürich, Forchstrasse 340, 8008 Zürich, Switzerland
}

male chiropractors [1]. However, this will likely be changing in the future in Switzerland as currently, at the chiropractic program in the faculty of medicine at this major university, the majority of chiropractic students are female. Therefore a change in the sex distribution in the chiropractic profession in Switzerland is likely in the future. The data obtained in the Swiss Job Analysis study further shows some differences in practice characteristics between male and female chiropractors in Switzerland in terms of hours worked per week and number of patients treated per week. As the percentage of female chiropractors in Switzerland will rise in the future according to the 
sex ratio in the chiropractic education program, this could lead to a shortage of Swiss chiropractors in the future as women are more likely to work part time $[1,5-8]$. In the general population, neck pain caused by musculoskeletal problems is extremely common $[9,10]$. After low back pain, neck pain and its associated disability is the second most common chief complaint that leads to patients seeking chiropractic care $[1,11-13]$. Previous research has already revealed predictors of positive outcome in neck pain patients, such as a short duration of neck pain as well as neck stiffness [14-16]. Reported prior improvement for both acute and chronic patients is also discovered to be a predictor of improvement in neck pain patients [16]. However, to date there has been no research on chiropractic treatment in neck pain patients investigating if the sex of the chiropractor is related to outcomes., This is relevant as at times there has been the misconception that at least some of the chiropractic treatments require a certain degree of strength to perform properly and that female chiropractors may find this more challenging compared to their male colleagues. Conversely, some patients comment that they prefer a female chiropractor as they assume her treatment may be gentler.

As the complaint of neck pain is common in the general population and as the percentage of female chiropractors is increasing in Switzerland, the hypothesis is that there is no difference in treatment outcomes in neck pain patients based on the sex of the chiropractor. Therefore, purpose of this study was to evaluate if the outcomes of neck pain patients treated by female chiropractors are the same as the outcomes of neck pain patients treated by male chiropractors.

\section{Methods}

\section{Study design}

This is a secondary analysis of data from a prospective cohort outcomes study [16] with follow-up time points of 1 week, 1 month, 3 months, 6 months and 1 year. Ethics approval for this study was obtained from the Canton of Zürich Switzerland ethics committee (EK-19/2009) and written informed consent was obtained from all patients.

\section{Patients}

For this study, successive new patients were recruited from numerous chiropractic practices in Switzerland. The patients were over the age of 18, suffered from neck pain of any duration and had not received any chiropractic or manual therapy in the previous 3 months. Excluded were patients with specific pathologies of the cervical spine which are contraindications to chiropractic manipulative therapy. These included acute fractures, infections, tumours, inflammatory arthropathies, Paget's disease, anti-coagulation therapy, cervical spondylotic myelopathy, known unstable congenital anomalies and severe osteoporosis [13].

All 260 members of the Association of Swiss Chiropractors were asked to recruit patients for this study and all examinations and treatments occurred in the participating chiropractic practices. All chiropractors received notification and instructions about this study plus the study protocol by email. Shortly before starting the data collection, details about the study were also discussed verbally at the annual mandatory post-graduate continuing education convention and any questions from the chiropractors were answered. At this information meeting as well as via email it was additionally emphasized that the participating chiropractors should not change their treatment methods, due to the fact that the purpose of this study was to evaluate outcomes as they were normally found in chiropractic practices. Therefore it was not desired to standardize neither the treatment method nor the treatment number. Nevertheless, it is known that most of the chiropractic patients in Switzerland (76-100\%) are at least treated with the diversified technique [1].

\section{Baseline pain and disability data}

Immediately before starting the treatment, every patient had to rate the severity of their current neck pain using the numerical pain rating scale (NRS) and a separate NRS for arm pain. The NRS is a scale evaluating the intensity of pain over the past $24 \mathrm{~h}$, ranging from 0 (no pain) to 10 (worst pain imaginable).

Additionally, the Bournemouth Questionnaire for neck (BQN) disability, which has been validated and translated into German [17], was used for the baseline information. In each practice the questionnaires were distributed by the office staff. The BQN is a short-form multidimensional instrument and includes questions on pain, disability and also psychosocial topics. Altogether the BQN covers 7 domains by using an 11-point numerical rating scale (from 0 to 10) to evaluate each domain separately. These 7 domains include: (I) pain; (II) physical function (disability in activities of daily living (ADL)); (III) disability in social activities; (IV) anxiety; (V) depression; (VI) work-related fear avoidance (at home and at the workplace); and (VII) pain locus of control. In addition to the score obtained for each domain (with a maximum of 10 points each), the total score of all domains together (maximal 70 points) was also calculated. It has been shown, that the German version of the $\mathrm{BQN}$ is more responsive to change than the German versions of the Neck Pain and Disability (NPAD) questionnaire and the Neck Disability Index (NDI) for all scales [17]. Each of the 7 items of the BQN was designed to be used individually as well as the total score $[18,19]$. 


\section{Clinical and demographic baseline data}

At the first consultation, additional information was collected by the treating chiropractor and sent via fax to the university chiropractic research assistant. This supplementary data was completed in a baseline information form and included: patient age, sex, marital status, paid employment, whether or not the onset of pain was due to trauma, whether or not the patient smokes, whether or not the patient was currently taking pain medication, number of previous episodes, duration of current complaint, whether or not the patient had signs and symptoms of cervical radiculopathy, whether or not the patient complained of additional dizziness and the patient's general state of health. This data was used in the original predictors of outcome paper [16]. Only patient age, sex, duration of complaint, medication use and the presence of radiculopathy were included in this secondary analysis of outcomes based on the sex of the treating chiropractor.

\section{Outcome measures}

To evaluate how much the patients 'improved' overall compared to their condition prior to treatment at the various outcome time points, the Patient's Global Impression of Change (PGIC)-scale was used [20, 21]. The PGIC-scale is a 7-point rating scale to report the patient's improvement from just prior to the start of treatment to the current time point ranging from 1 (much better) to 7 (much worse). Scores of 1 (much better) or 2 (better) on the PGIC-scale were categorized as 'improved' (primary outcome), whereas scores of 3 (slightly better), 4 (no change), 5 (slightly worse), 6 (worse) and 7 (much worse) were defined as 'not improved' [17]. A score of 5, 6 or 7 was additionally categorized as worsening (secondary outcome). This scoring system has been used in other studies [16, 22].

Additional outcomes were assessed using the NRS (neck) and the BQN scores at the follow up time points and their subscale change (baseline minus 1 week, 1 month, 3 months, 6 months or 1 year) scores (secondary outcomes).

The outcome data collection (1 week, 1 month, 3 months, 6 months and 1 year) after the start of the treatment was done by research assistants at the university hospital who were unknown to the patients. Via telephone interviews to the patients, they collected the data of the NRS neck pain, NRS arm pain, Patient Global Impression of Change (PGIC) scale and the $\mathrm{BQN}$. The patients knew that they were enrolled in the outcomes study, having signed informed consents. This occurred at the point of data collection for the original outcomes study, and therefore patients were not nformed that subsequent analysis would include comparing treatment outcomes between male and female chiropractors.

\section{Statistical analysis}

Baseline factors with categorical variables were compared between patients of male and female chiropractors using the Chi-square test. For continuous variables (i.e. change scores/normally distributed data) the unpaired Student's ttest was used. To analyze differences within patient groups for continuous variables, the paired t-test was performed. The proportion of patients reporting 'improvement' or 'worsening' was compared between the sexes of the chiropractors for each data collection time point using the Chi-square test. The NRS and BQ actual and change scores were compared between the patients of male and female chiropractors at each time point using the unpaired Student's t-test. SPSS version 21.0 (IBM, Armonk, New York) was used for the statistical analysis.

\section{Results}

\section{Baseline characteristics}

Of the 260 active members of the Association of Swiss Chiropractors, 78 contributed patients to this study (30\% response rate), with 46 chiropractors being male and 32 being female. As a total of 70 of the 260 Swiss chiropractors were female and 190 were male, this corresponds to a $45.7 \%$ response rate for the female chiropractors and a $24.2 \%$ response rate for the male chiropractors. Baseline data was provided for 849 neck pain patients with 450 being treated by male and 399 being treated by female chiropractors.

Overall there were more women $(65 \%, N=551)$ than men (35\%) suffering from neck pain in this study. Additionally, there was a statistically significant difference in the proportion of male versus female patients treated when comparing the sexes of the chiropractors. Female chiropractors treated significantly more female $(71.2 \%)$ than male $(28.8 \%)$ neck pain patients compared to male chiropractors $(59.3 \%$ female and $40.7 \%$ male patients) $(p=0.0001)$. At baseline, neck pain patients of male chiropractors were significantly older than patients treated by female chiropractors $(p=0.0001)$ (Table 1$)$ and they reported statistically significantly higher levels of neck pain compared to the patients of their female co-workers $(p=0.012)$ (Table 1). All scores on the Bournemouth Questionnaire at baseline were compared between the two groups, but there were no significant differences.

There was also an association between patients diagnosed with radiculopathy and consulting a female chiropractor. A higher proportion of patients seeing female chiropractors presented with the clinical signs and symptoms of radiculopathy $(17.3 \%)$ compared to the patients of male chiropractors $(11.2 \%)(p=0.014)$ (Table 1$)$.

Comparing the duration of symptoms of the two groups showed that the percentage of acute and chronic patients was distributed fairly equally between male and 
Table 1 Baseline Characteristics

\begin{tabular}{|c|c|c|c|}
\hline & Male DCs & Female DCs & $p$-value \\
\hline \multirow[t]{2}{*}{ Patient sex } & $40.7 \%$ male $(n=183)$ & $28.8 \%$ male $(n=115)$ & $0.0001^{*}$ \\
\hline & $59.3 \%$ female $(n=267)$ & $71.2 \%$ female $(n=284)$ & \\
\hline Patient age (y), (SD) & 43.15 (SD 13.659) & 39.71 (SD 13.637) & $0.0001^{*}$ \\
\hline Radiculopathy present & $11.2 \%(n=49)$ & $17.3 \%(n=69)$ & $0.014^{*}$ \\
\hline Pain medication use & $27 \%(n=121)$ & $35 \%(n=140)$ & $0.046^{*}$ \\
\hline Chronicity < 4 weeks (acute) & $44.9 \%(n=197)$ & $44.1 \%(n=174)$ & $0.02^{*}$ \\
\hline Chronicity 4-8 weeks (subacute) & $8.9 \%(n=39)$ & $12.9 \%(n=51)$ & \\
\hline Chronicity 8-12 weeks (subacute) & $11.4 \%(n=50)$ & $6.1 \%(n=24)$ & \\
\hline Chronicity > 12 weeks (chronic) & $34.9 \%(n=153)$ & $37.0 \%(n=146)$ & \\
\hline Baseline NRS (SD) & 5.951 (SD 2.211) & 5.561 (SD 2.256) & $0.012^{*}$ \\
\hline BQ1 (pain) & 5.680 (SD 2.262) & 5.651 (SD 2.306) & 0.853 \\
\hline BQ2 (disability in ADL) & 4.185 (SD 2.823) & 3.999 (SD 2.764) & 0.337 \\
\hline BQ3 (disability in social activities) & 3.614 (SD 3.120) & 3.433 (SD 3.079) & 0.400 \\
\hline BQ4 (anxiety) & 5.382 (SD 2.883) & 5.344 (SD 2.827) & 0.851 \\
\hline BQ5 (depression) & 3.441 (SD 3.099) & 3.376 (SD 3.066) & 0.762 \\
\hline BQ6 (work realted fear avoidance) & 4.442 (SD 2.936) & 4.577 (SD 3.064) & 0.515 \\
\hline BQ7 (pain locus of contro|\}) & 5.035 (SD 2.864) & 4.916 (SD 2.846) & 0.548 \\
\hline Baseline BQ total (SD) & 31.761 (SD 15.124) & 31.319 (SD 15.396) & 0.676 \\
\hline
\end{tabular}

${ }^{*} p \leq 0.05, \mathrm{n}=$ number of patients, $S D$ standard deviation, $y$ year, NRS numerical rating scale, BQ Bournemouth Question, $D C$ Doctor of Chiropractic. Italic and bold $=p<0.05$

female chiropractors. A significant difference in chronicity was only found in the two subgroups of the subacute category, with male chiropractors having more patients suffering from neck pain at 8-12 weeks, but having less patients complaining of neck pain at $4-8$ weeks $(p=0.02)$ (Table 1$)$.

\section{Differences in patients reporting 'improvement' or 'worsening' treated by male or female chiropractors}

A significant difference in the percentage of patients reporting clinically relevant 'improvement' was discovered at 1 month, where the patients of the female chiropractors did significantly better $(p=0.035)$ (Table 2$)$. At 1 week, 3 months, 6 months and 1 year, there were no significant differences in the outcomes between the two groups (Table 2).

Comparing reported 'worsening' between the two groups revealed a significant difference in worsening at 3 months. A higher proportion of patients treated by male chiropractors $(4.8 \%)$ reported worsening compared to patients treated by female chiropractors $(1.2 \%)$. At all the other time points, no significant difference in worsening between the two groups could be detected (Table 2).

\section{Secondary outcome differences}

One month after the start of treatment, patients treated by male chiropractors reported significantly higher scores on the BQN for pain, disability in activities of daily living, social disability, pain locus of control, BQN total score as well as in the NRS for neck pain compared to the patients of female chiropractors (Table 3). The same results were found at the follow-up time point of 3 months (Table 3). At 6 months, the BQN total score was almost significant $(p=0.051)$ (Table 3$)$.

Table 2 Comparison of proportion of patients 'improved', 'not improved' and 'worse' based on PGIC responses

\begin{tabular}{lllll}
\hline & PGIC & Male DC's & Female DC's & $p$-value \\
\hline At 1 week & improved & $56.5 \%$ & $54.6 \%$ & 0.863 \\
& not improved & $43.5 \%$ & $45.4 \%$ & \\
& worse & $4.3 \%$ & $4.2 \%$ & 1.000 \\
At 1 month & improved & $69.2 \%$ & $76.7 \%$ & $\mathbf{0 . 0 3 5 ^ { * }}$ \\
& not improved & $30.8 \%$ & $23.3 \%$ & \\
& worse & $3.8 \%$ & $1.8 \%$ & 0.161 \\
At 3 months & improved & $74.9 \%$ & $81.4 \%$ & 0.053 \\
& not improved & $25.1 \%$ & $18.6 \%$ & \\
& worse & $4.8 \%$ & $1.2 \%$ & $\mathbf{0 . 0 1 2}$ \\
At 6 months & improved & $78.0 \%$ & $78.6 \%$ & 0.840 \\
& not improved & $22.0 \%$ & $21.4 \%$ & \\
& worse & $2.9 \%$ & $2.2 \%$ & 0.710 \\
At 1 year & improved & $81.3 \%$ & $80.6 \%$ & 0.957 \\
& Not improved & $18.7 \%$ & $19.4 \%$ & \\
& worse & $3.3 \%$ & $2.7 \%$ & 1.000 \\
\hline${ }^{*} p \leq 0.05, P G I C$ Patient's Global Impression of Change, DC Doctor of Chiropractic. \\
Italic and bold $=$ & $p<0.05$ & & &
\end{tabular}


Table 3 Comparing NRS- and BQN-Scores at 1 week, 1 month, 3 months, 6 months and 1 year between male and female Chiropractors

\begin{tabular}{|c|c|c|c|}
\hline & $\begin{array}{l}\text { Patients of Male } \\
\text { DCs mean (SD) }\end{array}$ & $\begin{array}{l}\text { Patients of Female } \\
\text { DCs mean (SD) }\end{array}$ & $p$-value \\
\hline At 1 week NRS neck score & 3.628 (SD 2.265) & 3.407 (SD 2.335) & 0.222 \\
\hline At 1 week BQ1 (pain) & 4.529 (SD 2.160) & 4.517 (SD 2.252) & 0.943 \\
\hline At 1 week BQ2 (disability in ADL) & 2.983 (SD 2.629) & 2.978 (SD 2.740) & 0.984 \\
\hline At 1 week BQ3 (disability in social activities) & 2.298 (SD 2.857) & $2.472(\mathrm{SD} 3.047)$ & 0.455 \\
\hline At 1 week BQ4 (anxiety) & 4.052 (SD 2.701) & 3.924 (SD 2.799) & 0.554 \\
\hline At 1 week BQ5 (depression) & 2.081 (SD 2.638) & 2.175 (SD 2.796) & 0.657 \\
\hline At 1 week BQ6 (work-related fear avoidance) & 3.529 (SD 2.874) & 3.709 (SD 3.035) & 0.446 \\
\hline At 1 week BQ7 (pain locus of control) & 4.571 (SD 3.023) & 4.518 (SD 3.081) & 0.828 \\
\hline At 1 week $B Q$ total score & 24.060 (SD 13.172) & 24.357 (SD 14.309) & 0.783 \\
\hline At 1 month NRS neck score & 2.879 (SD 2.249) & 2.442 (SD 2.146) & $0.008^{*}$ \\
\hline At 1 month BQ1 (pain) & 3.043 (SD 2.175) & 2.720 (SD 2.135) & $0.046^{*}$ \\
\hline At 1 month BQ2 (disability in ADL) & 1.787 (SD 2.375) & 1.330 (SD 2.135) & $0.007^{*}$ \\
\hline At 1 month BQ3 (disability in social activities) & 1.348 (SD 2.384) & $1.003(\mathrm{SD} 2.131)$ & $0.044^{*}$ \\
\hline At 1 month BQ4 (anxiety) & 2.572 (SD 2.736) & 2.240 (SD 2.579) & 0.099 \\
\hline At 1 month BQ5 (depression) & 1.401 (SD 2.352) & 1.156 (SD 2.206) & 0.156 \\
\hline At 1 month BQ6 (work-related fear avoidance) & $2.630($ SD 2.741) & $2.301(\mathrm{SD} 2.745)$ & 0.115 \\
\hline At 1 month BQ7 (pain locus of control) & 3.516 (SD 3.197) & 3.033 (SD 3.263) & $0.048^{*}$ \\
\hline At 1 month $\mathrm{BQ}$ total score & 16.324 (SD 13.742) & 13.765 (SD 13.148) & $0.012^{*}$ \\
\hline At 3 months NRS neck score & 2.402 (SD 2.308) & 1.866 (SD 2.084) & $0.002^{*}$ \\
\hline At 3 months BQ1 (pain) & 2.562 (SD 2.403) & 2.016 (SD 2.105) & $0.002^{*}$ \\
\hline At 3 months BQ2 (disability in ADL) & $1.225(\mathrm{SD} 2.024)$ & 0.891 (SD 1.771) & $0.022^{*}$ \\
\hline At 3 months BQ3 (disability in social activities) & 0.762 (SD 1.781) & 0.483 (SD 1.486) & $0.027^{*}$ \\
\hline At 3 months BQ4 (anxiety) & 2.005 (SD 2.515) & 1.660 (SD 2.370) & 0.066 \\
\hline At 3 months BQ5 (depression) & 1.066 (SD 2.092) & 0.869 (SD 1.886) & 0.199 \\
\hline At 3 months BQ6 (work-related fear avoidance) & 1.858 (SD 2.535) & 1.964 (SD 2.694) & 0.601 \\
\hline At 3 months BQ7 (pain locus of control) & 2.787 (SD 3.043) & 2.266 (SD 2.789) & $0.021^{*}$ \\
\hline At 3 months BQ total score & 12.274 (SD 12.527) & 10.119 (SD 11.531) & $0.020^{*}$ \\
\hline At 6 months NRS neck score & 2.191 (SD 2.235) & 1.949 (SD 2.155) & 0.151 \\
\hline At 6 months BQ1 (pain) & 2.292 (SD 2.307) & 2.067 (SD 2.271) & 0.199 \\
\hline At 6 months BQ2 (disability in ADL) & 1.081 (SD 1.965) & 0.857 (SD 1.713) & 0.115 \\
\hline At 6 months BQ3 (disability in social activities) & 0.700 (SD 1.742) & 0.478 (SD 1.542) & 0.080 \\
\hline At 6 months BQ4 (anxiety) & 1.949 (SD 2.491) & 1.639 (SD 2.352) & 0.097 \\
\hline At 6 months BQ5 (depression) & 1.119 (SD 2.183) & 0.860 (SD 1.913) & 0.103 \\
\hline At 6 months BQ6 (work-related fear avoidance) & 2.045 (SD 2.735) & 1.747 (SD 2.532) & 0.147 \\
\hline At 6 months BQ7 (pain locus of control) & 2.511 (SD 2.956) & 2.197 (SD 2.832) & 0.162 \\
\hline At 6 months $\mathrm{BQ}$ total score & 11.675 (SD 12.739) & 9.822 (SD 11.956) & 0.051 \\
\hline At 1 year NRS neck score & 2.155 (SD 2.278) & 1.833 (SD 2.182) & 0.066 \\
\hline At 1 year BQ1 (pain) & 2.283 (SD 2.341) & 2.051 (SD 2.281) & 0.200 \\
\hline At 1 year BQ2 (disability in ADL) & 0.978 (SD 1.882) & 0.831 (SD 1.870) & 0.316 \\
\hline At 1 year BQ3 (disability in social activities) & 0.595 (SD 1.621) & 0.524 (SD 1.691) & 0.581 \\
\hline At 1 year BQ4 (anxiety) & 2.267 (SD 2.782) & 1.902 (SD 2.595) & 0.085 \\
\hline At 1 year BQ5 (depression) & 1.057 (SD 2.105) & 0.892 (SD 2.016) & 0.309 \\
\hline At 1 year BQ6 (work-related fear avoidance) & 1.892 (SD 2.652) & 1.763 (SD 2.609) & 0.534 \\
\hline At 1 year BQ7 (pain locus of control) & 2.231 (SD 2.825) & 2.017 (SD 2.709) & 0.330 \\
\hline At 1 year BQ total score & $11.322(S D$ 12.542) & 9.967 (SD 12.503) & 0.167 \\
\hline
\end{tabular}

${ }^{*} p \leq 0.05, S D$ standard deviation, NRS numerical rating scale, BQ Bournemouth Question, $D C$ Doctor of Chiropractic, $A D L$ activities of daily living. Italic and bold $=p<0.05$ 
The change scores were used to assess how much the $\mathrm{BQN}$ and NRS neck scores changed from baseline to the various outcome time points between the two groups. Only the change scores of the time points in which there was a significant difference between the two groups in the NRS and BQN scores above were assessed (1 month and 3 months). By 1 month after the start of treatment, no significant difference was found in the change scores between the two groups treated by male or female chiropractors, although the BQ6 evaluating the change in work-related fear avoidance was almost significant $(p=0.052)$ (Table 4). At 3 months, the change score for $\mathrm{BQ1}$ (pain) was significantly higher within patients of female chiropractors, indicating a higher amount of neck pain reduction.

\section{Discussion}

As the purpose of this study was to compare treatment outcomes in Swiss neck pain patients treated by male versus female chiropractors, it was reassuring, both for patients and clinicians, to find that the neck pain patients treated by female chiropractors reported a significantly higher proportion of patients 'improved' only at the 1 month outcome time point compared to patients treated by their male colleagues. At the later data collection time points there were no significant differences in the percentage of patients reporting 'improvement' between male and female chiropractors, although the PGIC-result at 3 months was almost significant in favor of female chiropractors' patients. However, there was a significant difference in the proportion of patients reporting 'worsening' at the 3 month time point with patients treated by male chiropractors being 4 times more likely to report 'worsening' (i.e. $4.8 \%$ of patients) compared to patients treated by female chiropractors (i.e. $1.2 \%$ of patients). Both of these values are very low however compared to the proportion of 'improved' patients for both groups of clinicians.

The same supporting results were found in the BQand NRS neck data where patients of female chiropractors had better scores at 1 month and also at 3 months. Contributing to the significant difference in outcome of the NRS neck data at 1 and 3 months in favor of female chiropractors may be the fact that the NRS neck pain score was significantly higher in male chiropractors' patients at baseline and also continued to be significantly higher after 1 and 3 months of treatment. This suggests that the pain reduction within male and female chiropractor's patients over time was most likely similar considering that patients of male chiropractors presented with higher levels of pain. Indeed, the fact that the NRS change scores at 1 and 3 months did not show any significant differences between patients of male and female chiropractors supports this conclusion.

Table 4 Comparing NRS- and BQ-change-scores from baseline to 1 and 3 months between male and female chiropractors

\begin{tabular}{|c|c|c|c|}
\hline & $\begin{array}{l}\text { Patients of male } \\
\text { DCs mean (SD) }\end{array}$ & $\begin{array}{l}\text { Patients of female } \\
\text { DCs mean (SD) }\end{array}$ & $p$-value \\
\hline 1 month NRS neck change score & 3.079 (SD 2.936) & 3.077 (SD 2.636) & 0.993 \\
\hline 1 month BQ1 change score (pain) & $2.593(\mathrm{SD} 2.867)$ & 2.898 (SD 2.699) & 0.147 \\
\hline 1 month BQ2 change score (disability in ADL) & 2.262 (SD 3.249) & 2.621 (SD 2.803) & 0.118 \\
\hline 1 month BQ3 change score (disability in social activities) & 2.140 (SD 3.446) & 2.493 (SD 3.138) & 0.158 \\
\hline 1 month BQ4 change score (anxiety) & $2.721(\mathrm{SD} 3.351)$ & 3.003 (SD 3.199) & 0.256 \\
\hline 1 month BQ5 change score (depression) & 1.954 (SD 2.924) & 2.139 (SD 2.926) & 0.404 \\
\hline 1 month BQ6 change score (work-related fear avoidance) & 1.714 (SD 3.424) & 2.214 (SD 3.336) & 0.052 \\
\hline 1 month BQ7 change score (pain locus of control) & 1.536 (SD 3.878) & $1.893(\mathrm{SD} 4.061)$ & 0.237 \\
\hline 1 month $\mathrm{BQ}$ total change score & 14.912 (SD 17.481) & 17.277 (SD 16.445) & 0.065 \\
\hline 3 month NRS neck change score & 3.519 (SD 2.982) & 3.614 (SD 2.787) & 0.667 \\
\hline 3 month BQ1 change score (pain) & 3.102 (SD 3.094) & 3.564 (SD 2.712) & $0.040^{*}$ \\
\hline 3 month BQ2 change score (disability in ADL) & 2.893 (SD 3.336) & 3.073 (SD 3.057) & 0.463 \\
\hline 3 month BQ3 change score (disability in social activities) & 2.848 (SD 3.347) & 3.011 (SD 3.187) & 0.517 \\
\hline 3 month BQ4 change score (anxiety) & 3.367 (SD 3.472) & 3.623 (SD 3.250) & 0.322 \\
\hline 3 month BQ5 change score (depression) & 2.353 (SD 3.055) & 2.458 (SD 3.016) & 0.652 \\
\hline 3 month BQ6 change score (work-related fear avoidance) & $2.542(\mathrm{SD} 3.437)$ & 2.475 (SD 3.485) & 0.803 \\
\hline 3 month BQ7 change score (pain locus of control) & 2.290 (SD 3.894) & 2.673 (SD 3.840) & 0.201 \\
\hline 3 month $\mathrm{BQ}$ total change score & 19.415 (SD 17.930) & 20.945 (SD 16.829) & 0.252 \\
\hline
\end{tabular}

${ }^{*} p \leq 0.05, S D$ standard deviation, NRS numerical rating scale, $B Q$ Bournemouth Question, $D C$ Doctor of Chiropractic, $A D L$ activities of daily living. Italic and bold $=p<0.05$ 
As mentioned above, 4 subscales and the total score of the Bournemouth Questionnaire (pain, disability in ADL, disability in social activities, pain locus of control) also showed significantly lower scores in patients seen by female chiropractors compared to those seen by male chiropractors after 1 and 3 months. However, a look at the actual change scores of the BQ-data does not confirm significant differences between male and female chiropractors' patients over time. Although statistically significant differences were found for several of the outcome measures between the patients of male and female chiropractors, when looking at the actual numerical differences, it could be argued that they are not clinically relevant. Other than the BQ total scores, the actual numerical differences were less than 0.6 of an NRS point. With large sample sizes such as those in this study, it is not difficult to find statistically significant differences. However, authors and readers must be cautious about implying too much clinical relevance in such cases when there is less than a 1 point difference on the NRS scale.

It has been suggested that the change in pain over time may be more relevant to the patient and gives a better clinical picture than the pain level at a single point in time [23]. This suggests that the change scores and the PGIC-results are more meaningful in this study than the BQ- and NRS actual neck results at the various time points. Therefore, the most important results are the significant 'improvement' after 1 month followed by the significant BQ1 change score after 3 months of treatment, both representing better findings within female chiropractors' patients. Nevertheless, all scores 6 months and 1 year after the start of treatment were similar in patients seen by male and female chiropractors.

Patient management not only consists of good manual treatments, but the communication style plays a conceivably important role as well. Doctor-patient communication may be an additional and important factor in order to get a good and satisfying result at the end of a treatment period. It has been shown that female physicians view doctor-patient communication in a more positive way, that they talk more often and longer to patients and that they set a higher value on psychosocial aspects, confidence and empathy when talking to patients $[24,25]$. This may be a reason why female chiropractors achieved slightly better results in their neck pain patients after 1 month. But as the satisfaction was very high not only within female, but also within male chiropractors' patients at all other time points, good care seems to be provided by both genders.

The baseline data showed that consulting a chiropractor because of neck pain was more common in women than men (65\% versus $35 \%)$. This result is supported by other studies which report that in general predominantly women suffer from neck pain $[10,23,25,26]$. The fact that women tend to have smaller and weaker neck muscles compared to men, but not a comparatively smaller or lighter head is a plausible explanation for a higher amount of vulnerability of female necks and therefore neck pain found in the literature [27].

Additionally, a strong relationship between patient sex and chiropractor sex was revealed in this study. Although more females than males suffered from neck pain, female chiropractors still had a significantly higher percentage of female patients than male chiropractors. Previous studies already investigated if there is a correlation between patient and doctor sex and discovered a "gender concordance preference" in females [28-31]. One reason why women often tend to consult a female rather than a male physician is the opinion that doctors of the same gender are more empathic and easier to talk to. This makes the patient feel more comfortable and at ease during the case history and especially during the examination [28]. Particularly for health professions dealing with more intimate and psychosocial health problems, "gender concordance preference" seems to play a major role [32]. As chiropractic is a profession with a lot of physical contact, it is very likely that some women prefer female chiropractors for the abovementioned reasons.

Some studies have indicated that not only in general but also with neck pain, women report significantly higher pain and disability levels than men [25, 27, 33]. On the other hand, a study by Peterson et al. showed no difference in pain levels between male and female neck pain sufferers [23] and in this current study the male patients presented with higher levels of pain. As there is little and inconclusive information about pain level differences in neck pain patients depending on their sex, it is not possible to identify if this has an effect on the outcome of this study.

Interestingly, the results showed that female chiropractors had significantly more patients diagnosed with radiculopathy. However, their outcomes were better in the short term. One low back pain study showed that chiropractic patients with low back pain and additional leg pain improved less than patients without leg pain [34], while other studies did not support this result $[35,36]$. Additionally, Swiss neck pain patients with arm pain undergoing chiropractic treatment have been shown to improve as much as patients without arm pain [16]. This suggests that the significant difference in radiculopathy between patients of male and female chiropractors in this present study did not have an influence on the better outcomes of female chiropractors' patients in the short term.

Whether or not neck pain is of a long or short duration has been shown to have an impact on the treatment outcome. While patients suffering from acute neck 
pain present with higher pain and disability levels, they improve faster and in a higher proportion than chronic neck pain sufferers, although many chronic neck pain patients improve as well $[14,16]$. Thus a shorter duration of neck pain at baseline has been shown to result in a more favorable outcome [14]. This has also been found in cervical disc herniation patients undergoing chiropractic treatment in Switzerland [37]. However, the significance shown in the chronicity categories of this present study did not seem to have an impact on the outcome as the proportion of acute and chronic patients was the same for male and female chiropractors. Although the results showed a significant difference in the distribution of the acute, subacute and chronic patients between patients seen by male and female chiropractors, this significance is of minor importance as it only involved the two subacute classifications which had the least number of patients overall.

Pain medication use in this present study was more common in patients of female chiropractors at baseline. This is explained by the findings that female chiropractors are visited more commonly by female rather than male neck pain sufferers. As stated by several studies, women use significantly more prescription and non-prescription analgesics than men [38-40]. This may also have contributed to the differences in baseline pain levels reported in this current study where the male patients reported higher pain scores. Because of the correlation between women and use of analgesics reported in the mentioned studies it can be suggested that the higher percentage of female patients in female chiropractors' practices is the reason why female chiropractors have more patients using analgesics. To date there are no studies investigating if the additional use of analgesics leads to a better outcome in chiropractic patients than chiropractic treatment alone. Thus it cannot be clarified if the higher amount of analgesics use in female chiropractors' patients influences the outcome positively.

It is possible that the age of patients has an influence on the outcome with older patients improving less than younger patients. Female chiropractors have significantly younger patients than male chiropractors and it is possible that this would be related to the better outcome in patients of female chiropractors. However, the actual numeric age difference between patients seen by male and female chiropractors is small as the large sample size in this study may have made this significant. Thus, the age difference in this study also should not impact the outcome relevantly.

It was interesting to observe that there was a higher response rate from female chiropractors compared to males contributing patients to this study. This is likely due to the fact that female Swiss chiropractors are more likely to work part time compared to their male colleagues
[1]. No information is available comparing characteristics between chiropractors who contributed patients to this study and those who did not.

\section{Limitations}

As this study was not a randomized controlled trial but a prospective cohort study, no control or comparison group was included. Therefore, whether the improvement that occurred in both study groups is mainly a result of the chiropractic treatment or only a consequence of the natural history of healing cannot be verified. Chiropractors from all parts of Switzerland contributed patients to this study. These patients chose their own chiropractor so randomization was not possible in this setting. Therefore, it is likely that inequalities between the two study groups additionally influenced the results. By analyzing the unbalanced baseline data and their possible impact on the results in the discussion section, possible additional sources of influence were identified, but some may have remained undetected.

In this study, data collection at baseline was conducted with a paper questionnaire and the follow-up data was collected by telephone interviews. It has been shown that using telephone interviews for data collection has a slight positive effect on the results, as patients tend to report better scores when answering the questions by telephone than in a written form [41-43]. By using research assistants for the telephone interviews who were unknown to patients and chiropractic practices, an attempt was made to minimize this effect. Furthermore, anonymity was ensured.

As there was no standardization of the treatment method desired in this study and as the chiropractors did not have to describe their treatment procedures, it is unknown which treatment method was used by how many chiropractors and if one procedure achieved better results than others. Whether male and female chiropractors in this study used the same or different treatment methods could therefore also not be determined. Nevertheless, from the results of the Swiss Job Analysis 2009 it can be assumed that the vast majority of the patients in this study were treated with at least the diversified technique [1].

The participating chiropractors contributed different numbers of patients to this study, as the number of patients was not determined. Therefore, chiropractors contributing numerous patients to this study did have a higher impact on the overall outcome than chiropractors contributing only few patients to this study. Additionally, the number and frequency of treatments was not determined. This decision was left to the chiropractor, as the purpose of this study was to evaluate outcomes as they would be found routinely in chiropractic practice.

Thirty percent of chiropractors (78 of 260) in Switzerland contributed patients to this present study. Whether or not 
these $30 \%$ truly represented the average of Swiss chiropractors remains unknown.

\section{Conclusions}

The results showed that the sex of the chiropractor was only linked to the treatment outcomes of these chiropractic neck pain patients at the 1 month time point. Additionally, although the BQ neck pain change score at 3 months also favored the female chiropractors, the sample size was very large in this study and the actual numerical differences were comparatively small, though statistically significant. Therefore these neck pain change scores at 3 months are not likely to be clinically relevant. Nevertheless, this study has shown that with their treatment of neck pain patients, female chiropractors achieve at least equally satisfying results as their male colleagues. This suggests that the quality of the chiropractic treatment of neck pain patients in Switzerland will not change in the future despite a prospective sex shift in the chiropractic profession.

\section{Abbreviations \\ ADL: Activities of daily living; BQ: Bournemouth Questionnaire; BQ1: Bournemouth Question 1; BQN: Bournemouth Questionnaire for Neck Pain; DC: Doctor of Chiropractic; NDI: Neck Disability Index; NPAD Scale: Neck Pain and Disability Scale; NRS: Numerical pain rating scale; PGIC Scale: Patient's Global Impression of Change Scale}

\section{Acknowledgements}

Not applicable.

\section{Funding}

The European Academy for Chiropractic, the Unisciencia foundation and the Balgrist Hospital Stiftung provided funding for this study.

\section{Availability of data and materials}

The SPSS data set is available through the Chiropractic Medicine Department, Orthopaedic University Hospital, University of Zürich, Switzerland.

\section{Authors' contributions}

JT: Literature search, data analysis and interpretation of data, drafting and revising the manuscript. CP: Ethics approval submission, data entry, data interpretation, revising the manuscript, final approval of the manuscript. BKH: Concept and design of the study, manuscript review and final approval.

\section{Ethics approval and consent to participate}

Ethics approval for this study was obtained from the Canton of Zürich Switzerland ethics committee (EK-19/2009) and written informed consent was obtained from all patients.

\section{Consent for publication}

Not applicable.

\section{Competing interests}

The authors declare that they have no competing interests.

\section{Publisher's note}

Springer Nature remains neutral with regard to jurisdictional claims in published maps and institutional affiliations.
Received: 21 March 2017 Accepted: 12 July 2017

Published online: 17 July 2017

\section{References}

1. Humphreys BK, Peterson CK, Muehlemann D, Haueter P. Are Swiss chiropractors different than other chiropractors? Results of the job analysis survey 2009. J Manip Physiol Ther. 2010;33:519-35.

2. Christensen MG, Kollasch MW, Ward R, et al. Job analysis of chiropractic 2005: a project report, survey analysis, and summars of the practice of chiropractic within the United states. National Board of Chiropractic Examiners: Greeley (Colo; 2005.

3. http://www.nightingale-collaboration.org/images/Consulting_the_Profession_ A_Survey_of_UK_Chiropractors_2004.pdf.

4. Pollentier A, Langworthy J. The scope of chiropractic proactice: a survey of chiropractors in the UK. Clin Chiropr. 2007;10:147-55.

5. Weaver AC, Wetterneck TB, Whelan CT, Hinami K. A matter of priorities? Exploring the persistent gender pay gap in hospital medicine. J Hosp Med. 2015;10:486-90

6. Tandjung R, Senn O, Marty F, Krauss L, Rosemann T, Badertscher N. Career after successful medical board examination in general practice-a cross-sectional survey. Swiss Med Wkly. 2013;143:W13839.

7. McMurray JE, Linzer M, Konrad TR, Douglas J, Shugerman R, Nelson K. The work lives of women physicians: results from the physician work life study. J Gen Intern Med. 2000;15:372-80.

8. Buddeberg-Fischer B, Stamm M, Buddeberg C, Bauer G, Hämmig O, Knecht M, et al. The impact of gender and parenthood on physicians' careers professional and personal situation seven years after graduation. BMC Health Serv Res. 2010;10:40.

9. Bovim G, Schrader H, Sand T. Neck pain in the general population. Spine. 1994;19:1307-9.

10. Fejer R, Kyvik KO, Hartvigsen J. The prevalence of neck pain in the world population: a systematic critical review of the literature. Eur Spine J. 2006;15:834-48.

11. Ailliet L, Rubinstein SM, de Vet HC. Characteristics of chiropractors and their patients in Belgium. J Manip Physiol Ther. 2010;33:618-25.

12. Hartvigsen J, Sorensen LP, Graesborg K, Grunnet-Nilsson N. Chiropractic patients in Denmark: a short description of basic characteristics. J Manip Physiol Ther. 2002;25:162-7.

13. Rubinstein S, Pfeifle CE, van Tulder MW, Assendelft WJ. Chiropractic patients in the Netherlands: a descriptive study. J Manip Physiol Ther. 2000:23:557-63.

14. Rubinstein SM, Knol DL, Leboeuf-Yde C, de Koekkoek TE, Pfeifle CE, van Tulder MW. Predictors of a favorable outcome in patients treated by chiropractors for neck pain. Spine. 2008;33:1451-8.

15. Thiel HW, Bolton JE. Predictors for immediate and global responses to chiropractic manipulation of the cervical spine. J Manip Physiol Ther. 2008;31:172-83.

16. Peterson C, Bolton J, Humphreys BK. Predictors of outcome in neck pain patients undergoing chiropractic care: comparison of acute and chronic patients. Chiropr Man Therap. 2012;20:27.

17. Soklic M, Peterson C, Humphreys BK. Translation and validation of the German version of the Bournemouth questionnaire for neck pain. Chiropr Man Therap. 2012;20:2.

18. Bolton JE, Breen AC. The Bournemouth questionnaire: a short-form comprehensive outcome measure. I. Psychometric properties in back pain patients. J Manip Physiol Ther. 1999;22:503-10.

19. Bolton JE, Humphreys BK. The Bournemouth questionnaire: a short-form comprehensive outcome measure. II. Psychometric properties in neck pain patients. J Manip Physiol Ther. 2002;25:141-8.

20. Bolton JE. Sensitivity and specificity of outcome measures in patients with neck pain: detecting clinically significant improvement. Spine. 2004;29:2410-7.

21. Newell D, Bolton JE. Responsiveness of the Bournemouth questionnaire in determining minimal clinically important change in subgroups of low back pain patients. Spine. 2010;35:1801-6.

22. Humphreys BK, Peterson C. Comparison of outcomes in neck pain patients with and without dizziness undergoing chiropractic treatment: a prospective cohort study with 6 month follow-up. Chiropr Man Therap. 2013;21:3.

23. Peterson CK, Humphreys BK, Hodler J, Pfirrmann CW. Gender differences in pain levels before and after treatment: a prospective outcomes study on 3,900 Swiss patients with musculoskeletal complaints. BMC Musculoskelet Disord. 2012;13:241. 
24. Löffler-Stastka H, Seitz T, Billeth S, Pastner B, Preusche I, Seidman C. Significance of gender in the attitude towards doctor-patient communication in medical students and physicians. Wien Klin Wochenschr. 2016;128:663-8.

25. Leresche L. Defining gender disparities in pain management. Clin Orthop Relat Res. 2011;469:1871-7.

26. Bot SD, van der Waal JM, Terwee CB, van der Windt DA, Schellevis FG, Bouter LM, et al. Incidence and prevalence of complaints of the neck and upper extremity in general practice. Ann Rheum Dis. 2005;64:118-23.

27. Peterson C, Bolton J, Wood AR, Humphreys BK. A cross-sectional study correlating degeneration of the cervical spine with disability and pain in United kingdom patients. Spine. 2003;28:129-33.

28. Bishop FL, Smith R, Lewith GT. Patient preferences for technical skills versus interpersonal skills in chiropractors and physiotherapists treating low back pain. Fam Pract. 2013;30:197-203.

29. Bishop FL, Massey Y, Yardley L, Lewith GT. How patients choose acupuncturists: a mixed-methods project. J Altern Complement Med. 2011;17:19-25.

30. Ahmad F, Gupta H, Rawlins J, Stewart DE. Preferences for gender of family physician among Canadian European-descent and south-Asian immigrant women. Fam Pract. 2002;19:146-53.

31. Fang MC, MCCarthy EP, Singer DE. Are patients more likely to see physicians of the same sex? Recent national trends in primary care medicine. Am J Med. 2004;117:575-81.

32. Kerssens JJ, Bensing JM, Andela MG. Patient preference for genders of health professionals. Soc Sci Med. 1997:44:1531-40.

33. Ruau D, Liu LY, Clark JD, Angst MS, Butte AJ. Sex differences in reported pain across 11,000 patients captured in electronic medical records. J Pain. 2012;13:228-34.

34. Axen I, Jones JJ, Rosenbaum A, Lovgren PW, Halasz L, Larsen K, et al. The Nordic back pain subpopulation program: validation and improvement of a predictive model for treatment outcome in patients with low back pain receiving chiropractic treatment. J Manip Physiol Ther. 2005;28:381-5.

35. Peterson CK, Bolton J, Humphreys BK. Predictors of improvement in patients with acute and chronic low back pain undergoing chiropractic treatment. J Manip Physiol Ther. 2012;35:525-33.

36. Leboeuf-Yde C, Gronstvedt A, Borge JA, Lothe J, Magnesen E, Nilsson O, et al. The nordic back pain subpopulation program: demographic and clinical predictors for outcome in patients receiving chiropractic treatment for persistent low back pain. J Manip Physiol Ther. 2004;27:493-502.

37. Peterson CK, Schmid C, Leemann S, Anklin B, Humphreys BK. Outcomes from magnetic resonance imaging-confirmed symptomatic cervical disk herniation patients treated with high-velocity, low-amplitude spinal manipulative therapy: a prospective cohort study with 3-month follow-up. J Manip Physiol Ther. 2013;36:461-7.

38. Dale O, Borchgrevink PC, Fredheim OM, Mahic M, Romundstad P, Skurtveit S. Prevalence of use of non-prescription analgesics in the Norwegian HUNT3 population: impact of gender, age, exercise and prescription of opioids. BMC Public Health. 2015;15:461

39. Paulose-Ram R, Hirsch R, Dillon C, Losonczy K, Cooper M, Ostchega Y. Prescription and non-prescription analgesic use among the US adult population: results from the third National Health and nutrition examination survey (NHANES III). Pharmacoepidemiol Drug Saf. 2003;12:315-26.

40. Antonov Kl, Isacson DG. Prescription and nonprescription analgesic use in Sweden. Ann Pharmacother. 1998;32:485-94.

41. Hays RD, Kim S, Spritzer KL, Kaplan RM, Tally S, Feeny D, et al. Effects of mode and order of administration on generic health-related quality of life scores. Value Health. 2009;12:1035-9.

42. Powers JR, Mishra G, Young AF. Differences in mail and telephone responses to self-rated health: use of multiple imputation in correcting for response bias. Aust N Z J Public Health. 2005:29:149-54.

43. Hanmer J, Hays RD, Fryback DG. Mode of administration is important in US national estimates of health-related quality of life. Med Care. 2007:45:1171-9.

\section{Submit your next manuscript to BioMed Central and we will help you at every step:}

- We accept pre-submission inquiries

- Our selector tool helps you to find the most relevant journal

- We provide round the clock customer support

- Convenient online submission

- Thorough peer review

- Inclusion in PubMed and all major indexing services

- Maximum visibility for your research

Submit your manuscript at www.biomedcentral.com/submit
) Biomed Central 